\title{
Avaliação do processo de descentralização das ações de saúde
}

\author{
Evaluating health care decentralization
}

Ligia Maria Vieira da Silva 1

\begin{abstract}
The aim of this paper is to discuss a framework for health service evaluation related to the decentralization process in Brazil. Methodological and conceptual issues related to the evaluation steps proposed are analysed: a) contextual analysis; b) strategic analysis; $c$ ) management characteristics; d) coverage and effectiveness monitoring; and, e) implementation analysis. Problems and advantages related to an articulated approach between external perspective and empowerment evaluation are discussed. Counties's classification in stages of the decentralization process is analysed related with existing proposals for health services reform. The achievement of theoretical, technical and political consensus is highlighted as a need for transforming evaluation in a tool for the management process.
\end{abstract}

Key words Evaluation; Decentralization; Health Policy
Resumo O presente artigo discute estratégias para a avaliação do processo de descentralização das ações de saúde no Brasil a partir de revisão da literatura sobre a temática. É feita a discussão sobre as indicações, possibilidades e problemas metodológicos relativos aos momentos e atributos propostos para avaliação: a) análise de contexto; b) análise estratégica; c) características da gestão; d) monitoramento da cobertura e da efetividade de problemas priorizados; e, e) análise de implantação. As vantagens e os problemas da articulação entre os enfoques externo e interno ou de auto-avaliação são discutidos. A classificação dos municípios segundo estágios do processo de descentralização é analisada tendo por referência propostas atuais de reorganização das práticas de saúde. A formação de consenso teórico, técnico e politico entre os atores sociais envolvidos com o processo de descentralização é identificada como componente necessário para assegurar a efetividade da avaliação e sua incorporação como instrumento de gestão.

Palavras-chave Descentralização; Municipalização; Avaliação

\footnotetext{
1 Instituto de Saúde Coletiva da Universidade Federal da Bahia Rua Padre Feijó 29, Canela 40110-170, Salvador Bahia, Brasil ligiamvs@ufba.br
} 


\section{Introdução}

Após a promulgação da Constituição de 1988, a principal ênfase das políticas públicas de saúde no Brasil tem-se relacionado com a municipalização das ações e serviços de saúde. Situado oficialmente como "o caminho" para a reorganização dos serviços de saúde no país, a partir da IX Conferência Nacional de Saúde (Brasil, 1992) o processo de descentralização hoje atinge 5.049 municípios brasileiros, o que corresponde a 91,7\% do total (Brasil, 1998) e requer o desenvolvimento de estratégias para a sua avaliação. Grande parte da bibliografia disponível sobre a avaliação, e particularmente sobre a avaliação em saúde, refere-se à avaliação tecnológica (Power et al., 1994), ou de programas (Hartz et al., 1997), ora enfatizando a qualidade (Donabedian, 1988), ora as avaliações econômicas de custo-efetividade (Banta \& Luce, 1993), recorrendo para essa finalidade tanto a técnicas quantitativas (Cook et al., 1982) quanto qualitativas (Guba \& Lincoln, 1988). A diversidade do instrumental metodológico atualmente disponível para a avaliação permite focalizar não apenas práticas, serviços e programas, mas abordar totalidades mais complexas, como sistemas nacionais e locais de saúde. Algumas das proposições elaboradas com essa finalidade correspondem a uma listagem de possíveis indicadores (OMS, 1990), à definição de momentos (Campoamor, 1990), ou têm enfocado especificamente componentes da gestão e organização dos serviços (Heimann et al., 1998). Conquanto essas proposições correspondam a avanços e auxiliem no equacionamento do problema da avaliação sistêmica, diversas lacunas persistem decorrentes das tensões existentes entre o todo e as partes, entre o nacional e o local, e entre os componentes universais e os contextuais. Nessa perspectiva, o presente artigo pretende contribuir para com o desenvolvimento de estratégias para a avaliação de sistemas de saúde a partir das ferramentas metodológicas e técnicas disponíveis, tomando como objeto o processo de descentralização das ações de saúde no Brasil.

\section{O objeto da avaliação: a municipalização da saúde}

Inicialmente cabe situar o objeto da avaliação, ou seja, o município com suas novas atribui- ções constitucionais. Cabe ao município, de forma autônoma, porém em articulação com as demais esferas de governo, formular políticas locais de saúde e gerenciar os recursos e as instituições voltadas para a prestação de atenção à saúde (Carvalho \& Santos, 1992). Além disso, o município deve reorganizar serviços e práticas de saúde na perspectiva de um novo modelo assistencial capaz de controlar danos, riscos e causas dos problemas priorizados pelos atores sociais envolvidos (Paim, 1993). Essa pode ser considerada como a imagem-objetivo proposta na NOB/96, que corresponderia à Gestão Plena do Sistema Municipal (Brasil, 1997).

Embora o município passe a assumir autonomia em relação a determinadas funções, esta é relativa tendo em vista a necessidade de articulação nacional das diversas instituições municipais e estaduais num sistema único de saúde conforme está proposto na Constituição Federal. Essa necessidade não é dada por razões meramente formais, e sim tendo em vista a existência de problemas de saúde comuns a diversos municípios e estados onde a adoção de estratégias de controle pactuadas pode potencializar o efeito das mesmas, bem como a necessidade de apoio técnico e institucional dos níveis mais estruturados para os menos estruturados. Por esse motivo, a manutenção de sistemas de informação, tanto de mortalidade quanto de morbidade nacional, é um pré-requisito para o acompanhamento e a avaliação dessas experiências municipais a partir de critérios e padrões que permitam comparações na medida do possível.

\section{Estratégias}

Entendendo estratégia como forma de implementação de uma política (Testa, 1995), quer dizer, os modos de aproximação dos objetivos e as formas de superação dos obstáculos, a definição de estratégias de avaliação corresponderia a um conjunto de opções relacionadas com a seleção de questões, métodos e técnicas para a identificação de critérios, indicadores e padrões, definição do universo do estudo, formas de análise, seqüência dos momentos, perspectiva do avaliador, enfim, dos desenhos possíveis em cada situação.

Em se constituindo o município como uma totalidade complexa onde o campo da saúde possui como qualquer outro campo uma au- 
tonomia relativa (Bourdieu, 1989), ou seja, diversos processos decorrem de lógicas internas ao campo relativas aos seus componentes (instituições e atores), porém ao mesmo tempo encontra-se intimamente vinculado a outros campos e particularmente ao campo do poder, a avaliação deverá contextualizar e descrever de que forma as diversas redes de relações constitutivas desses campos interferem com as práticas de saúde. O que corresponde a indagar acerca do significado local do campo e a contextualizar a situação sob análise. Em que consiste o "sistema” de saúde do município em questão? Trata-se de um conjunto de estabelecimentos públicos municipais? Qual o papel das instituições federais e estaduais? Quais as características do setor privado? Por outro lado, a compreensão do campo requer saber "quem é quem" neste sistema? Quais as características e como estão distribuídas as diversas formas de poder - técnico, administrativo e político? Quais são os bens materiais e simbólicos que estão em jogo no campo e que implicam acumulações sociais?

O núcleo de referência para a avaliação são os problemas de saúde (danos, riscos e determinantes) definidos e priorizados pelos atores sociais que fazem parte do campo e que dessa forma constituem-se na segunda indagação do processo avaliativo de ordem genérica e pontual. O próprio processo de definição dos problemas pode revelar características da gestão e do planejamento que nortearão o desenvolvimento subseqüente da avaliação. Esta será conduzida por um especialista externo à instituição, adotará uma perspectiva de auto-avaliação ou se constituirá numa articulação entre ambas as abordagens? Em seguida, é o momento da análise estratégica, quando cabe indagar acerca da pertinência e adequação entre problemas, objetivos e intervenções propostas (Campoamor, 1990; Contandriopoulos et al., 1997).

A depender do resultado dos primeiros passos do processo avaliativo, algumas características ou atributos dos problemas priorizados poderão constituir-se em objeto de monitoramento, e outras características deverão ser matéria de estudos pontuais ou mesmo de investigações avaliativas. Propõe-se como atributos fundamentais para o monitoramento a cobertura e a efetividade das ações desenvolvidas para o enfrentamento dos problemas priorizados. A identificação de fenômenos paradoxais, tais como elevada cobertura e baixa efetividade ou simplesmente a existência de efeitos inesperados, pode ser utilizada como indicadores da necessidade de aprofundamento do processo de acompanhamento aqui denominado "sintonia fina". Corresponde à abertura da "caixa preta", ou seja, à realização de análises de implantação (Denis \& Champagne, 1997), a avaliações de qualidade (Donabedian, 1988), e aos estudos acerca da percepção dos usuários (Lemme et al., 1991).

\section{As perspectivas da avaliação}

O desenvolvimento de uma avaliação implica freqüentemente em escolhas relativas a perspectivas ou postos de avaliação. Ou seja, deverá a avaliação ser conduzida por um especialista externo à instituição que está sendo analisada, ou será preferível desencadear um processo interno de auto-avaliação? Essas duas dimensões traduzem, por vezes, não somente divergências metodológicas e teórico-conceituais, mas também têm claras implicações políticas e gerenciais. A avaliação externa freqüentemente tem sido associada à idéia de objetividade e tem sido acompanhada pela explicitação prévia de critérios, padrões e indicadores, bem como pela utilização de desenhos controlados, experimentais ou observacionais com elevado grau de validade interna e externa (Cook et al., 1982). A adoção dessa abordagem permitiria ao avaliador evitar os diversos viéses possíveis além de propiciar a distância necessária à objetividade requerida por um julgamento isento (Scriven, 1997). No extremo oposto, a partir da crítica à abordagem objetivista, alguns autores propõem estratégias não-estruturadas, em que todos os momentos e componentes da avaliação são definidos em negociação entre o facilitador e os sujeitos para os quais a avaliação se destina. Várias são as denominações desse enfoque: empowerment evaluation, que pode ser traduzido como "avaliação que autofortalece” (Fetterman, 1996), avaliação de quarta geração ou construtivista (Guba \& Lincoln, 1988). As vantagens dessa segunda alternativa estariam na possibilidade de contextualizar as avaliações, de ampliar a compreensão do significado das práticas através da apreensão das representações dos atores sociais, além do envolvimento dos mesmos no processo de identificação de problemas e modificação de suas condições de vida a partir de um processo desalienante. Essas oposi- 
ções entre o objetivo e o subjetivo, o qualitativo e o quantitativo, decorrem da influência exercida por diferentes correntes sociológicas sobre o campo da avaliação.

Contudo, alguns autores defendem abordagens integradoras onde o método e as técnicas relacionam-se com o objeto da avaliação, sendo que em muitas circunstâncias uma combinação entre as diversas perspectivas não somente é possível, mas também desejável (Cook, 1997:48). No caso do objeto em questão - o processo de municipalização da saúde - as próprias atribuições constitucionais das diferentes esferas de governo impõem uma abordagem articulada entre a perspectiva externa e a interna. O Ministério da Saúde e as Secretarias Estaduais passam a ter atribuições fundamentalmente normativas e reguladoras, o que remete ao papel de avaliador externo. Dessa perspectiva, por exemplo, para o monitoramento de atributos-chaves nos municípios é necessário o aperfeiçoamento dos sistemas de informação nacionais e estaduais através da incorporação das variáveis necessárias. O desenvolvimento e a utilização de tipologia para a classificação dos municípios em estágios distintos, referentes à mudança do modelo assistencial, requerem a deflagração de um processo cujo âmbito é necessariamente estadual e nacional. Por fim, o monitoramento de problemas de relevância regional priorizados no Plano Estadual de Saúde ou outros selecionados, como eventos-sentinela, ou ainda segundo a técnica dos traçadores (Kessner et al., 1992), constitui-se em atribuição estadual.

Por outro lado, as novas responsabilidades municipais com a gestão do sistema de saúde requerem a incorporação da avaliação como componente do processo de planejamento, como atividade capaz de subsidiar as tomadas de decisão e como elemento auxiliar nas iniciativas voltadas para a mudança do modelo assistencial. $\mathrm{O}$ desenvolvimento de estratégias de auto-avaliação pode responder a essas necessidades contribuindo para que as informações geradas no processo de avaliação sejam realmente utilizadas para a gestão dos serviços. A incorporação da avaliação como uma atividade sistemática ao interior da gestão municipal de saúde pode ser considerada como um dos indicadores de modificação do modelo assistencial. Além disso, a auto-avaliação tem a vantagem de incorporar os atores sociais na reflexão sobre o significado de seu traba- lho, podendo constituir-se em atividade relevante para elevar a motivação para com o mesmo (Fetterman, 1996).

\section{A análise estratégica}

Após a contextualização da situação, a indagação seguinte recai sobre a pertinência e adequação entre problemas, objetivos e intervenções, o que corresponde a realizar análise estratégica e de intervenção (Campoamor, 1990; Contandriopoulos et al., 1997). Esse é um dos componentes que apresenta dificuldades para os gestores reconhecerem como necessária a avaliação; dado que requer uma postura autocrítica acerca do próprio trabalho de planificação. Contudo, sua realização pode trazer à tona elementos importantes acerca do processo de gestão, bem como da incorporação de inovações. Ao se indagar sobre o processo de definição de prioridades, as características do planejamento vão emergir. Ao interrogar sobre a adequação das atividades programadas aos problemas priorizados, sobre a viabilidade dos objetivos e metas, bem como sobre a eficácia das ações propostas, os gestores estarão revelando em que medida o município está assumindo, de fato, as rédeas do processo de formulação e condução das políticas de saúde, e não apenas possuindo planos formais e conselhos cartoriais.

\section{Monitoramento e avaliação pontual}

Muito já foi dito sobre as múltiplas definições existentes sobre avaliação e noções correlatas (Vieira da Silva \& Formigli, 1994; Contandriopoulos et al., 1997). Por esse motivo, cabe explicitar qual o sentido dado aos conceitos. Avaliação será usada aqui num sentido amplo que diz respeito a um julgamento sobre práticas ou objetos. Ela pode variar desde uma apreciação norteada pelo senso comum e assistemática até uma investigação avaliativa orientada por métodos e técnicas científicas, podendo ser pontual e localizada ou pode corresponder a um acompanhamento sistemático. Nesse último caso, a atividade será denominada monitoramento, que corresponde a uma avaliação sistemática sobre algumas características das práticas ou objetos, no caso do presente artigo, sobre o processo de descentralização das ações de saúde. 
As características ou atributos das instituições ou do processo a serem selecionados para o monitoramento deverão relacionar-se com os problemas priorizados pelos atores do município e também com aqueles de importância estadual e nacional. As características ou atributos aqui mencionados são aqueles que revelam aspectos relevantes das intervenções: a) relacionados com a disponibilidade e distribuição social dos recursos (cobertura, acesso e eqüidade); b) relacionados com o efeito das ações (eficácia, efetividade e impacto); c) relacionados com os custos (eficiência); d) relacionados com a adequação das ações ao conhecimento tecnológico disponível (qualidade técnico-científica); e) relacionados com a percepção dos usuários (satisfação, aceitabilidade) (Vieira da Silva \& Formigli, 1994). Como na prática é inviável monitorar todos esses atributos para os problemas priorizados, propõe-se selecionar inicialmente dois, considerados como nucleares: a cobertura e a efetividade. É evidente que a seleção poderia ser outra a depender de especificidades locais bem como do estágio de desenvolvimento do processo de descentralização. Contudo, tendo em vista a especificidade do objeto, onde as ações de saúde podem passar a ser implementadas de forma intersetorial, tendo em vista a natureza da determinação dos problemas de saúde das populações,o monitoramento da abrangência das ações ou sua cobertura passa a ser pré-requisito para o acompanhamento de sua efetividade.

Os atributos do sistema selecionados para o monitoramento evidentemente irão requerer a produção de informação de forma sistemática e contínua, ou seja, deverão ser incorporados ao sistema de informação do município.

\section{O monitoramento da cobertura}

A cobertura corresponde à proporção da população alvo que se beneficia de determinada intervenção (Rossi \& Freeman, 1993), ou ao grau de adequação entre as necessidades e a oferta de ações de saúde. A distinção feita por Soberón entre cobertura potencial e cobertura real é de grande utilidade à medida que a primeira revela as possibilidades, enquanto a segunda resulta do trabalho efetivamente realizado (Soberón, 1988). Dessa forma, as discrepâncias verificadas entre as duas cobertu- ras são indicadoras de possíveis problemas na implementação das ações em questão.

Um problema importante relacionado com o cálculo da cobertura consiste nas estimativas populacionais no nosso meio nos anos intercensitários em decorrência dos fluxos migratórios internos e externos ao município. Por outro lado, a utilização de serviços de saúde freqüentemente escapa à definição administrativa de áreas de abrangência, e concentra-se em alguns estabelecimentos e municípios por razões variadas que vão desde a maior acessibilidade até a qualidade do serviço prestado (Vieira da Silva et al., 1995). Por esses motivos, tem sido recomendado pela OMS a realização de inquéritos periódicos para o monitoramento das coberturas vacinais (Henderson \& Sundareson, 1982). A possibilidade de superestimação das coberturas calculadas a partir das informações rotineiras dos serviços tem sido evidenciada por inquéritos populacionais (Vieira da Silva et al., 1997).

Também o conceito de população alvo e da eficiência da cobertura está a requerer debate e explicitação por parte dos gestores. Qual a população alvo do SUS? É toda a população, ou é apenas a parcela de baixa renda? O SUS persiste como um projeto de atenção universal, ou corresponde a uma cesta básica para os pobres? Caberia utilizar o conceito de eficiência de cobertura que corresponde à proporção da população que realmente necessita do programa e o utiliza, retirada a proporção da população que não necessita porém utiliza? (Rossi \& Freeman, 1993). Essas são definições políticas com implicações técnicas nas estratégias e indicadores para o monitoramento da cobertura.

\section{O monitoramento da efetividade}

Aferir o efeito de ações de saúde em um sistema operacional, ou seja, avaliar a sua efetividade, constitui-se em um dos problemas mais complexos para a gestão municipal. Isto porque fenômenos sociais, culturais e psíquicos têm sido relacionados com a produção do processo saúde-doença, além da biologia humana e dos serviços de saúde (Possas, 1989; Evans et al., 1994; Castellanos, 1997). Por outro lado, do ponto de vista metodológico, a utilização do desenho experimental clássico, recomendado para estudos de avaliação de eficácia tecnológica, dificilmente pode ser utiliza- 
do em sistemas de saúde, principalmente por motivos éticos ou operacionais. Já os estudos epidemiológicos observacionais do tipo coorte e caso-controle podem ser usados para investigações avaliativas pontuais de efetividade, mas dificilmente podem constituir-se em estratégia para o acompanhamento da mesma em decorrência dos custos e problemas metodológicos e operacionais.

As possibilidades de monitoramento da efetividade em sistemas de saúde relacionamse principalmente com a análise de séries temporais de indicadores de morbi-mortalidade podendo apoiar-se num enfoque guiado pela teoria (theory-driven) baseado em estratégias de ampliação da base de evidências, tais como os desenhos sintéticos, aferição da causalidade através da identificação de padrões contextuais, do uso de técnicas qualitativas, da aferição de efeitos não previstos (Chen, 1990). Por outro lado, as pesquisas de síntese vêm sendo utilizadas como suporte às avaliações de efetividade em diversas circunstâncias, onde a realização de um estudo isolado não é capaz de responder a esse tipo de pergunta (Cook, 1997:48).

Outra possibilidade de abordagem do monitoramento da efetividade diante do importante papel atribuído às condições de vida tem sido a articulação entre o estudo de causas de morte evitáveis com a análise das desigualdades sociais. As causas evitáveis têm sido utilizadas como indicadores negativos, "eventossentinela”, capazes de monitorar o efeito e a qualidade dos serviços de saúde, principalmente a partir do trabalho coordenado por Rutstein, que, reunindo especialistas de diversas áreas, elaborou lista internacional de doenças preveníveis e (ou) evitáveis (Rutstein et al., 1976). Uma redução na mortalidade por causas evitáveis, superior àquela verificada por outras causas, foi relacionada com a efetividade dos serviços de saúde em seis países europeus, entre 1950 e 1980 (Charlton \& Velez, 1986), e em Québec, entre 1982 e 1990 (Pampalon, 1993). Na Suécia, uma investigação sobre a mortalidade por causas evitáveis, segundo grupos socioeconômicos, foi utilizada para a avaliação do efeito dos serviços de saúde (Westerling et al., 1996).

Nessa mesma direção, a Organização PanAmericana de Saúde (OPS) desenvolveu um indicador denominado "Brechas Redutíveis de Mortalidade", que mede o risco de morte atribuível às condições de vida (OPS/OMS, 1994).
Esse indicador, contudo, pode também revelar, indiretamente, a efetividade dos serviços de saúde quando se compara o comportamento da mortalidade por causas evitáveis entre regiões com características sociais semelhantes.

\section{A sintonia fina: análise de implantação, avaliação da qualidade e satisfação dos usuários}

A análise da situação de saúde a partir das informações produzidas durante o processo de monitoramento possivelmente indicará necessidades ulteriores para a avaliação. Para problemas onde os resultados não sejam aqueles esperados, ou, onde há discrepâncias entre as coberturas potencial e real ou entre a cobertura e os resultados, estão indicadas as análises de implantação, a avaliação da qualidade técnico-científica e da satisfação dos usuários. Ou seja, se quantitativamente a oferta de ações é suficiente porém os efeitos esperados não são observados, há que se investigar se as ações previstas foram implementadas adequadamente de acordo com o plano e quais os determinantes contextuais do grau de implantação encontrado (Denis \& Champagne, 1997), se existe conformidade das práticas com o conhecimento disponível e quais as percepções da população em relação às ações, ou seja, corresponde a abrir a "caixa preta" e tentar explicar os processos e suas razões.

As diversas questões propostas por Heimann e colaboradores para a avaliação do processo de descentralização, relacionadas com: 1) o processo de planejamento e gestão;2) o financiamento; 3 ) a gerência de recursos humanos; 4) a organização e oferta de serviços; e, 5) a participação comunitária ; podem fazer parte de um roteiro para auxiliar a análise de implantação (Heimann et al., 1998).

De particular importância se reveste a análise do contexto diante de variações na implantação e de efeitos inesperados. Às análises feitas sobre as características do campo seriam acrescidas outras relativas às diversas organizações componentes, tais como hospitais, centros de saúde, clínicas privadas e secretarias de governo envolvidas. Como estão distribuídas as diversas espécies de poder nessas instituições? Quais as características organizacionais? Quem são os principais atores e quais as relações estabelecidas entre eles? De que forma essas dinâmicas interferiram com o grau 
de implantação observado e os efeitos? A resposta a essas indagações requer freqüentemente a realização de investigações avaliativas que podem ser estudos de caso, estudos comparativos, ou estudos experimentais (Denis \& Champagne, 1997).

\section{Um possível ponto de partida}

A avaliação de sistemas de saúde requer idas e vindas entre enfoques extensivos, com análise de múltiplos atributos e indicadores correlatos e enfoques intensivos, em que alguns aspectos são selecionados para investigação em profundidade. $\mathrm{O}$ esforço de recuperar a totalidade e identificar as dimensões contextuais e históricas pode se acompanhar também de iniciativas relacionadas com a busca de características universais. Vista desse ângulo, a elaboração de uma tipologia para a análise do processo de descentralização das ações de saúde pode corresponder a uma objetivação necessária sem ser objetivista ou corresponder a uma visão reducionista do processo, dado que se constitui em parte da estratégia de avaliação, realizada em articulação com as análises anteriormente mencionadas. Justifica-se na medida em que as formulações referentes à mudança do modelo assistencial, embora presentes no discurso oficial, têm assumido significados diferentes para os diversos atores sociais (Oliveira, 1999).

Com esse objetivo, a metodologia usada pela OPS para a análise prospectiva do ensino médico pode ser útil (Ferreira, 1988). Em sendo feita essa opção, o processo de descentralização poderia ser classificado em: a) incipiente; b) intermediário; e, c) avançado, de acordo com o estágio de reorganização das práticas e mudança do modelo assistencial. Os momentos da avaliação anteriormente discutidos poderiam servir de referência para a seleção de critérios (Donabedian, 1981), a sa- ber: a) análise de contexto; b) análise estratégica; c) características da gestão; d) monitoramento da cobertura e da efetividade de problemas priorizados; e, e) análise de implantação.

A definição da imagem-objetivo norteadora dos estágios acima mencionados poderia ter como ponto de partida algumas das proposições existentes para a reorganização das práticas, tais como o Plano Diretor para o Desenvolvimento da Vigilância da Saúde no Estado da Bahia (PLANDEVISA), em que a mudança do modelo de atenção consistiria em:

a) intervenção sobre problemas de saúde;

b) problemas que requerem atenção e acompanhamento contínuos;

c) adoção do conceito de risco;

d) articulação entre ações promocionais, preventivas e curativas;

e) atuação intersetorial;

f) ação sobre o território;

g) intervenção sob a forma de operações.

(Bahia-Devisa, 1998)

A definição dessa imagem-objetivo, bem como do desenho estratégico para a avaliação sistêmica contudo, requer a criação de consenso, inicialmente entre experts, e, posteriormente, entre os gestores municipais e demais atores sociais envolvidos com o processo de descentralização. Isso porque, tendo em vista que a avaliação corresponde a um julgamento sobre uma dada intervenção; no caso de um município, o resultado desse processo pode assumir uma dimensão política relacionada com o sucesso ou não da gestão em foco. Por outro lado, no nível individual a avaliação das práticas corresponde a um julgamento sobre o trabalho de sujeitos concretos com implicações também evidentes de ordem pessoal, institucional e social. Portanto, a efetividade da presente proposição requer a construção de consenso teórico, técnico e político para que a avaliação possa tornar-se uma ferramenta da gestão impulsionadora de transformações. 


\section{Referências}

Banta HD, Luce BR 1993. Health Care Technology and its Assessment. Oxford University Press, London.

Bourdieu P 1989. O Poder Simbólico. Difusão Editorial/ Livraria Bertrand, São Paulo, Lisboa.

Campoamor NR 1990. Evaluación de los sistemas locales de salud, p. 463-467. In JM Paganini \& RC Mir (orgs). Los Sistemas Locales de Salud. Organización Panamericana de La Salud. Washington, D.C.

Carvalho GI, Santos L 1992. Sistema Único de Saúde. Comentários à Lei Orgânica da Saúde (Lei 8.080/90 e Lei 8.142/90). Hucitec, São Paulo.

Castellhanos PL 1997. Epidemiologia, salud pública, situación de salud y condiciones de vida. Consideraciones conceptuales, p. 137-160. In RB Barata (org.) - Condições de Vida e Situação de Saúde. Abrasco, Rio de Janeiro.

Charlton J, Velez R 1986. Some international comparisons of mortality amenable to medical intervention. British Medical Journal 292: 295-301.

Chen H 1990. Theory-Driven Evaluations. Sage Publications, Newbury Park.

Contandriopoulos A, Champagne F, Denis J, Pineault R 1997. Avaliação na Área de Saúde: Conceitos e Métodos, p. 29-48. In Avaliação em Saúde: dos Modelos Conceituais à Prática na Análise da Implantação de Programas. H. ZMA. Fiocruz, Rio de Janeiro.

Cook T, Cook F, Mark M 1982. Modèles experimentaux et quasi experimentaux en recherche évaluative: une introduction, p. 105-142. Introduction aux Méthodes de Recherche Évaluative. Le Presses de l'Úniversité de Laval. LRRL Québec.

Cook TD 1997. Lessons learned in evaluation over the past 25 years, p. 30-52. In E Chelimsky \& WR Shadish (eds.) - Evaluation for the 21st Centrury: a Handbook. Sage Publications. Thousand Oaks, London, New Delhi.

Denis J, Champagne F 1997. Análise da implantação de programas, p. 49-88. In Z Hartz (ed.) - Avaliação em Saúde: dos Modelos Conceituais à Prática na Análise da Implantação de Programas. Fiocruz, Rio de Janeiro.

Donabedian A 1981. Criteria, norms and standards: what do they mean? American Journal of Public Health 71: 409-412.

Donabedian A 1988. Explorations in Quality Assessment and Monitoring. Vol. I. The Definition of Quality and Approaches to Its Assessment. Health Administration Press, Ann Arbor.

Evans RG, Baren ML, Marmor TR 1994. Why are Some People Healthy and Others Not? The Determinants of Health of Population. Aldine de Gruyter, Marmor Editores.

Ferreira JR 1988. El analisis prospectivo de la educación médica en América Latina. Educación Médica y Salud 22(3): 242-367.

Fetterman DM, Kaftarian SJ, Wandersman A 1996. Empowerment Evaluation: Knowledge and Tools for SelfAssessment \& Accountability. Sage Publications, Thousand Oaks, London, New Delhi.

Guba E, Lincoln Y 1988. Fourth generation evaluation, p. 21-49, Cap. I. In The Coming of Age of Evaluation. Sage Publications, Newbury Park.
Hartz ZA, Champagne F, Contandrioupoulos AP, Leal MD 1997. Avaliação do programa materno-infantil: análise de implantação em sistemas locais de saúde no nordeste do Brasil, p. 89-131. In ZA Hartz (ed.) - Avaliação em Saúde: dos Modelos Conceituais à Prática na Análise da Implantação de Programas. Fiocruz, Rio de Janeiro.

Heimann LS et al. 1998. A Descentralização do Sistema de Saúde no Brasil: uma Proposta de Investigação sobre o Impacto de Políticas. Instituto de Saúde. Secretaria de Estado da Saúde, São Paulo.

Henderson R, Sundareson T 1982. Cluster sampling to assess immunization coverage: a review of experience with a simplified sampling method. Bullettin World Health Organ 60(2): 253-260.

Kessner M, Kalk E, Singer J 1992. Evaluación de la calidad de la salud por el método de los procesos trazadores, p. 555-563. In KL White (ed.) - Investigaciones sobre Servicios de Salud: una Analogía. OPAS. Publicación Científica 534. Washington, D.C.

Lemme A, Noronha G, Resende J 1991. A satisfação do usuário em hospital universitário. Revista de Saúde Pública de São Paulo 25(1): 41-46.

Ministério da Saúde 1992. Cadernos da Nona: Descentralizando e Democratizando o Conhecimento. Brasília, D.F.

Ministério da Saúde 1997. Norma Operacional Básica do Sistema Único de Saúde. NOB-SUS 96. Brasília, D.F.

Ministério da Saúde 1998. Descentralização: Situação Atual do Processo de Habilitação. Brasília, D.F. http:// www.saude.gov.br/descen/habilita.htm

Oliveira MCBA 1999. Avaliação dos Efeitos do PACS nas Práticas de Saúde: um Estudo de Caso. Instituto de Saúde Coletiva. Universidade Federal da Bahia, Salvador, $114 \mathrm{pp}$.

OMS - Organização Mundial de Saúde 1990. Guía para una estrategia de evaluación y garantía de calidad de los sistemas de atención de salud, p. 455-462. In JM Paganini \& RC Mir (orgs.). - Los Sistemas Locales de Salud. Organización Panamericana de la Salud, Washington, D.C.

OPAS/OMS - Organização Pan-Americana de Saúde/Organização Mundial de Saúde 1994. Las Condiciones de Salud en las Americas. Publicación Científica 524. II, $68 \mathrm{pp}$.

Paim JS 1993. A reorganização das práticas em distritos sanitários. In EV Mendes - Distrito Sanitário: o Processo Social de Mudança das Práticas Sanitárias do Sistema Único de Saúde. Hucitec/Abrasco, São Paulo, Rio de Janeiro.

Pampalon R 1993. Avoidable mortality in Québec and its regions. Social Science and Medicine 37(6): 823-831.

Possas C 1989. Epidemiologia e Sociedade: Heterogeneidade Estrutural e Saúde no Brasil. Hucitec, São Paulo.

Power EJ, Tunis SR, Wagner JL 1994. Technology assessment and public health. Ann Rev Public Health 15: 561-579.

Rossi PH, Freeman HE 1993. Program monitoring, p. 163-213. In Evaluation a Systematic Approach. Sage Publications, Beverly Hills.

Rutstein DD et al. 1976. Measuring the quality of medical care. The New England Journal of Medicine 294(11): 582-588. 
Secretaria da Saúde do Estado da Bahia 1998. Plano Diretor para o Desenvolvimento da Vigilância da Saúde no Estado da Bahia (Plandevisa). BahiaDevisa, Salvador.

Scriven M 1997. Truth and objectivity in evaluation, p. 477-500. In E Chelimsky \& WR Shadish (eds.) - Evaluation for the 21st Century: a Handbook. Sage Publications, Thousand Oaks, London, New Delhi.

Soberón G 1988. La extensión de cobertura de los servicios de salud. Gaceta Médica de México 124(5-6): 163-166.

Testa M 1995. Pensamento Estratégico e Lógica de Programação: o Caso da Saúde. Hucitec/Abrasco, São Paulo, Rio de Janeiro.
Vieira da Silva LM, Formigli VLA 1994. Avaliação em saúde: limites e perspectivas. Cadernos de Saúde Pública 10(1): 80-91.

Vieira da Silva LM et al. 1995. O processo de distritalização e a utilização de serviços de saúde: avaliação do caso de Pau da Lima. Cadernos de Saúde Pública 11(1): 72-84.

Vieira da Silva LM et al. 1997. Coberturas vacinais superestimadas?. novas evidências a partir do inquérito de Pau da Lima. Revista Panamericana de Salud Pública 1: 444-450.

Westerling R, Gulberg A, Androsen AM 1996. Socioeconomic differences in "avoiding mortality" in Sweden (1986-1990). Int J Ep 25(3): 560-567. 